to be successful, both need to work in collaboration to provide accessible and acceptable services.

\section{UG6 PLEASE DON'T TELL MY GP: PATIENTS' CONCERNS ABOUT THE SHARING OF INFORMATION BETWEEN SEXUAL HEALTH CLINICS AND GENERAL PRACTITIONERS (GPS)}

${ }^{1}$ Qiang Lu*, ${ }^{2}$ Emily Clarke, ${ }^{1,3}$ Raj Patel, ${ }^{1}$ Harriet Eatwell, ${ }^{1}$ Rohilla Maarij. ${ }^{1}$ School of Medicine, University of Southampton, Southampton, UK; ${ }^{2}$ Department of Sexual Health, Solent NHS Trust, St Mary's Community Health Campus, Portsmouth, UK; ${ }^{3}$ Department of Sexual Health, Solent NHS Trust, Royal South Hants Hospital, Southampton, UK

\subsection{6/sextrans-2016-052718.55}

Background/introduction At sexual health clinics, patients are asked for permission to contact them by a variety of methods. When patients who have opted-out of GP contact are found to have a sexually transmitted infection (STI) and cannot be contacted despite multiple attempts, a case-by-case decision is often made, regarding breaching the patient's permissions and contacting their GP.

Aim(s)/objectives To determine why some patients decline GP contact, and to assess their views on GP contact against their expressed wishes, in order to treat an STI, when a patient is unable to be contacted by other means.

Methods This was a prospective, qualitative, NRES-approved study involving 10 semi-structured interviews with patients attending a level 3 UK sexual health clinic who had declined GP contact.

Results Three key areas of concern were identified: potential negative implications of permanently recording sexual health problems on GP records, including the effect on future life insurance and job applications; concerns about receptionists in GP surgeries breaking confidentiality in the reception area and being judgmental; and patients' close relationship with their GP. However, 8/10 of those interviewed supported a breach of permissions by contacting their GP in order to treat an STI.

Conclusion With the increased involvement of GPs in delivering sexual health services in the UK, it is essential that action is taken to improve patients' confidence in confidentiality protections at their GP. Sexual health clinics should ensure they explain why GP contact may be required in order to potentially increase patients' willingness for this to occur.

\section{Section 5 Poster presentations}

\section{P001 IN 2015, MSM ACCESSING PEPSE IS SIGNIFICANTLY MORE ASSOCIATED WITH CLUB DRUG USE THAN 2013/2014}

${ }^{1}$ Zoe Ottaway*, ${ }^{2}$ Daniel Richardson. ${ }^{1}$ Maidstone and Tunbridge Wells NHS Trust, Maidstone, Kent, UK; ${ }^{2}$ Brighton and Sussex University Hospitals NHS Trust, Brighton, Sussex, UK

\subsection{6/sextrans-2016-052718.56}

Background/introduction Recreational drug (RD) use is increasing in men who have sex with men (MSM) and increases sexual risk taking behaviour and possibly increasing attendances for PEPSE.

Aim(s)/objectives To identify Club drug use during PEPSE attendances in MSM in 2013/4 compared to 2015.

Methods Review PEPSE (MSM) attendance during two 4-month periods: November 2013 to February 2014 and March 2015 to June 2015.

Results 152 MSM attended for PEPSE: 51 in 2013/4 and 101 in 2015. The median age was 31 (18-79) years. Documentation of Club drug use during PEPSE episode increased significantly from $27 / 51(53 \%)$ in $2013 / 14$ to $100 / 101$ (99\%) during 2015 ( $p<0.001)$. Club drug use during PEPSE episode increased significantly from $9 / 51(18 \%)$ in $2013 / 4$ to $41 / 101$ (41\%) in 2015 (OR 3.19, $\mathrm{p}<0.005)$. There were no significant changes in the Club drugs being used: gamma-Butyrolactone(GBL), Mephedrone and Crystal Meth being the most frequent reported.

Discussion/conclusion Episodes of unsafe sex leading to access of PEPSE appear to be more associated with club drug use in 2015 than in 2013/4 and our documentation of this has improved. Identification of club drug use in MSM is an important harm intervention.

\section{P002 IS ENQUIRY REGARDING ALCOHOL CONSUMPTION AND ALCOHOL REDUCTION ADVICE ACCEPTABLE TO SEXUAL HEALTH SERVICE USERS? A CROSS-SECTIONAL STUDY OF CLINIC ATTENDEES}

\footnotetext{
1,2Martyn Wood. 'Mid-Cheshire Hospitals NHS foundation Trust, Cheshire, UK; ${ }^{2}$ Royal Liverpool University Hospital, Liverpool, UK
}

10.1136/sextrans-2016-052718.57

Background/introduction Problem alcohol consumption is a major health problem in the UK. Alcohol assessment and behavioural advice or "brief interventions" are effective in decreasing alcohol intake in primary and secondary care but not in sexual health clinics.

Aim(s)/objectives We assessed sexual health service user views towards alcohol screening using a prospective cross-sectional survey to identify any themes, which limit acceptability of these methods.

Methods Age, gender, alcohol consumption measured by AUDIT-C score, and opinion towards 10 statements on alcohol screening within a sexual health clinic were assessed.

Results 462 surveys were returned. Respondents were 64\% female, $36 \%$ male. Most, $53.7 \%$, were aged $\geq 25$ years, the highest number of responses was received from those aged 20-24 (32.2\%), median age category was 25-29 years. The majority of respondents, $61.6 \%$ had hazardous alcohol consumption. Males had more positive AUDIT-C scores (indicating hazardous alcohol consumption) compared to females (75\% vs 54\%, p < 0.001 ). Those aged $<30$ had more positive AUDIT-C scores $(67.9 \%$ vs $32.1 \%, \mathrm{p}<0.001)$. Attitudes to alcohol assessment performed by sexual health practitioners were positive (range 91.1\%$74.5 \%$ favourable), responses were less favourable, becoming negative towards the appropriateness of the sexual health clinic as a screening venue (range 56.7\%-33.6\% favourable). Responses to 4 out of 10 opinion statements were related (multivariate regression model) to age or AUDIT-C score.

Discussion/conclusion Different strategies need to be explored within sexual health for alcohol consumption reduction 